\title{
La Sistematización investigativa de las experiencias: del baile de los que sobran a la fiesta de los que faltan
}

\author{
María Belén Ortega-Senet \\ Doctora en Antropología Urbana y Movimientos Sociales. Trabajadora Social \\ Universidad Católica de la Santísima Concepción. Concepción, Chile \\ https://orcid.org/0000-0001-5457-6847• mbortega@ucsc.cl
}

\section{Resumen}

Este artículo es una invitación a movernos juntos hacia la construcción de espacios creativos, expresivos y nutritivos de reflexión-acción, a través de una estrategia determinada, la que nos convoca ahora: la Sistematización de Experiencias. A esta estrategia le hemos dado también el apellido de investigativa, porque queremos posicionarla como una oportunidad de construir articulación teórica-práctica, con reales poderes para generar conocimiento situado, relevando saberes que germinan en las relaciones cotidianas de la praxis social. En el texto, revisaremos cómo desde la posición de subalternidad (el baile de los que sobran) vamos batiendo tierra para configurar nuevos escenarios y posibilidades en los que la Sistematización puede jugar papeles interesantes. Pretendemos provocar el deseo de seguir buscando, de no cejar en la exploración de alternativas que nos liberen, a quienes participamos en el Trabajo Social, de convertirnos en gobernados, en técnicos y en usuarios. Compartimos también una experiencia, una propuesta incipiente de Sistematización que nos está permitiendo quebrar algunos aparatajes en la formación de la profesión y en la forma de relacionarnos con los procesos de prácticas pre-profesionales.

Palabras clave: Sistematización de Experiencias; Subalternidad; Resistencias; Prácticas pre-profesionales.

Recibido: 12/09/2020 | Aprobado: 06/11/2020 | Publicado: 01/01/2021

c) (i) (2) Esta obra está bajo una Licencia Creative Commons Atribución-NoComercialCompartirIgual 4.0 Internacional.

Financiación o proveniencia del artículo: este artículo es fruto de la experiencia realizada en la Carrera de Trabajo Social de la UCSC de Chile, con las asignaturas de Diseño de Memoria de Sistematización Social y Memoria de Sistematización Social.

¿Cómo citar este artículo? / How to quote this article?

Ortega-Senet, M. B. (2021). La Sistematización investigativa de las experiencias: del baile de los que sobran a la fiesta de los que faltan. Prospectiva. Revista de Trabajo Social e intervención social, (31), 93114. doi: 10.25100/ prts.v0i31.10613. 
Ortega-Senet

\title{
Research Based Systematization of Experiences: from the Dance of Those Who Abound to the Party of Those Who are Scarce
}

\begin{abstract}
This article is an invitation to move together towards the construction of creative, expressive and nutritious spaces of reflection-action through a determined strategy: The Systematization of Experiences. We have added the label of research to the name of this strategy because we want to position it as an opportunity to build theoretical-practical articulation, with real powers to generate situated knowledge, revealing knowledge that germinates in the daily relations of social praxis. In the text, we review how, from a position of subalternity (the dance of those who abound), we battle to configure new scenarios and possibilities where systematization can play interesting roles. We intend to provoke in everyone involved in the field of Social Work the desire to continue searching, to not stop exploring alternatives that will free us, to become governed, technicians and users. We also share an experience, an incipient proposal of Systematization that is allowing us to break some of the barriers in the formation of the profession and in the way we relate to the processes of pre-professional practices.

Keywords: Systematization of Experiences; Subalternity; Resistance; Preprofessional practices.

Sumario: 1. Introducción, 2. Reflexiones teórico-conceptuales, 2.1 De la subalternidad o el baile de los que sobran, 2.2 De las resistencias creactivas o la fiesta de los que faltan, 2.3 De cómo intentar que el baile llegue a la Academia y no al revés, 3. De la experiencia de la UCSC con la Sistematización Investigativa de Experiencias, 4. De los aprendizajes de la propuesta, 5. Conclusiones, 6. Referencias bibliográficas.
\end{abstract}




\section{Introducción}

La comunidad no es un medio, sino un fin. Trabajar para conseguir la comunidad como ideal en términos igualitarios, comunicacionales y creativos. (Bermudo,

2001, p. 50)

Con Rosa María Cifuentes tenemos un pequeño ¿cuento?, ¿canto?, ¿historia? No sabría cómo llamarlo, que ideamos a partir de una frase dicha por una conocida académica en un congreso sobre investigación y Trabajo Social allá por el año 2017: “La Sistematización no está invitada al Baile", señaló refiriéndose al Baile como la investigación científica.

De ahí pensamos en hacer una especie de cuentito en el que hablábamos sobre bailes de salón, de máscaras, de un mundo donde hay trabajadoras sociales de la academia que hacen lo posible por ser integradas en un espacio al que no todos están invitados, y sobre cómo la sistematización de experiencias tiene su propia fiesta: el baile de los que sobran (Cifuentes-Gil y Pantoja-Kauffmann, 2019).

Pero al pensar en el baile de los que sobran no quisiera que el imaginario les llevara a una sala triste y desolada, donde los excluidos se buscan y consuelan en la tragedia de ser los habitantes de los bordes, de los márgenes, los olvidados. El imaginario que me gustaría evocar es el de otros bailes, donde todos pueden encontrarse, donde los que expulsados de los salones hacen piruetas y se divierten. Cuando comparamos la Sistematización de Experiencias con el baile de los que sobran aludimos a fanfarrias, verbenas, chirigotas y zapateos. Una fiesta compartida que se adueñe de las calles, los barrios, los programas y espacios donde trabajamos, sean como sean. Este pasacalle dicharachero (que también tiene esfuerzo y ensayos detrás, no se confundan), ve a la Academia no como un sitio inalcanzable, sino como un espacio que debería ser prolongación de los espacios sociales, donde las murgas podrían colarse alegremente.

En este artículo, se pretende hacer una incursión en el contexto actual del Trabajo Social y las posibilidades que emergen para seguir re-considerándonos continuamente a través de momentos conscientes y encuentros colectivos de reflexión. Hay aquí una apuesta por las fortalezas de la Sistematización Investigativa de Experiencias como articuladora de teoría y práctica desde el análisis profundo: lo colectivo, lo lúdico y lo festivo no implica superficialidad. Y cuando me refiero a teoría no se trata de un corpus generalizable y rígido, homogenizante y categórico:

Una teoría de la intervención se debe plantear como dinámica, viva, siempre alerta y polisémica, basada en un entendimiento de las necesidades, desigualdades y las acciones sociales desde la vivencia cotidiana, con la inclusión de sus protagonistas, donde la información está al servicio de las personas, los colectivos y los pueblos, para la garantía de los derechos políticos, civiles, sociales, culturales y ecológicos. (Ortega-Senet, 2015, p. 288) 
Para ir desarmando algunos prejuicios, hay que aclarar que la Sistematización de Experiencias no tiene el papel simple de rescatar experiencias compartidas de forma participativa solamente, porque también se trata de eso (Jara-Holliday, 2018); sino que tiene otras tareas más fundamentales y complejas que ya han proclamado varios activistas de la metodología (Cifuentes-Gil, 1999, 2015; Jara-Holliday, 2020; Torres-Carrillo, 2019):

a. Recuperar el saber y la importancia del pensamiento crítico compartido de la intervención social acogiendo a todos los protagonistas.

b. Ser capaces de crear un momento consciente en las personas que viven los procesos, para lograr identificar aquellas prácticas que están participando en la creación de estigmatización, desigualdades, exclusión, control social; es decir perpetuando el sistema neoliberal con sus injusticias y desigualdades.

c. Y, por último, pero no menos importante, desmontar la separación artificiosa entre los que saben y los que no saben, considerándonos a todos como parte de la experiencia de intervención social y, por tanto, como parte del proceso de significarla, analizarla y compartirla.

No deja de ser extraño que una profesión que lleva décadas trabajando las posibilidades de Reconceptualización, como el Trabajo Social, y que todavía continúa en ello (Abad-Miguélez y Martín-Aranaga, 2015; Bermúdez-Peña, 2012; Cifuentes-Gil, 2015; Muñoz-Arce, 2018); que viene esforzándose en una integración orgánica entre acción y reflexión, y marcando toda una línea crítica, siga mirando a la Sistematización de Experiencias con desconfianza.

Estoy convencida de que la Reconceptualización no termina nunca, porque no es un proceso acabado, sino activo y permanente: ipobre de la sociedad en la que sus profesiones y disciplinas no se cuestionen a sí mismas en el papel que cumplen en la estructura social y política! Pero para lograr eso, debemos aprender; aprender a cuestionar y cuestionarnos el papel que llevamos a cabo en nuestros espacios de trabajo. Y cuestionarse requiere de cierta preparación, por ello la academia tiene el deber de acoger opciones y formas de auto observación. 


\section{Reflexiones teórico-conceptuales}

\subsection{De la subalternidad o el baile de los que sobran}

Sin desmedro de todo tipo de Baile, todos los espacios son bienvenidos; considerar a la Sistematización de Experiencias en el baile de los subalternos, es procurar no perder el Sur (como diría Mikjail). Tampoco queremos recrearnos en esa posición de los márgenes. Hay que tener vivo el ánimo, siempre, de deconstruir la estructura de posiciones en las que se ha puesto al Trabajo Social como subalterna; pero no podemos obviar cuáles son esas reglas y cómo se configura un campo de juego disciplinar, en el que solemos perder.

Esta subalternidad de la práctica del Trabajo Social ante otras disciplinas está muy relacionada con la visión técnica de nuestro quehacer, con el eclecticismo que la inunda (a veces sin sentido, a veces estupendamente), y con la invisibilidad del conocimiento adquirido a través de la experiencia que se desarrolla en la mayor parte de los espacios profesionales. Pero ¿por qué? ¿Por qué al Trabajo Social se le reconoce por estar en permanente contradicción teórico-práctica? ¿Por qué no terminamos de desasirnos de esta carga de complejos a pesar de llevar décadas (en Chile nada menos que 9 décadas) desarrollándose disciplinalmente? La respuesta está muy ligada a cómo la naturaleza del Trabajo Social ha sido apropiada, secuestrada, por el sistema neoliberal para constituirla como dispositivo discursivo y de control (García-Martín, 2017; Harris, 2014; Muñoz-Arce y Pantazis, 2019; Saavedra-Vásquez, 2018). Y también está conectada a cómo nos limitamos a trabajar en nuestros pequeños espacios de forma intuitiva, otra mecánica, desligándonos del necesario acto de consciencia crítica (Gianna y Mallardi, 2011; Hicks, 2016).

En el sistema político económico en el que nos movemos todos y todas, es la verdad económica la que rige la razón gubernamental; esto es, el mercado y las relaciones económicas son los que tienen "la razón" respecto a la política gubernamental (Foucault, 2009) y se constituye como La Racionalidad. El salto desde esa "racionalidad" a la tecnificación de nuestra labor es bastante simple. Las prácticas se han sometido a la lógica solicitada de estandarización y de eficacia técnica, asumiendo que el "éxito" de una política reside en el logro de objetivos institucionales, en desmedro de los objetivos o intereses de los participantes de los programas, y de los objetivos de transformación en sus dimensiones éticas y políticas (Ortega-Senet, Gómez-Fernández \& Tierney, 2020; Vivero-Arriagada, 2017).

Dentro de esta lógica impera la idea de que todo debe ser homogenizado, y de hecho se homogeniza. De este modo, en el Capitalismo Mundial Integrado, como dirían Guattari y Rolnik, (1999), los políticas y estrategias que se hacen en una parte del mundo (generalmente Europa y USA) sirven para otros territorios, porque la lógica que hay detrás es una, la única, la técnico-racional. Las explicaciones de los problemas también sirven para cualquier espacio-tiempo, y digo espacio y tiempo, porque hay algunas justificaciones 
Ortega-Senet

teóricas de intervención que pueden permanecer vigentes por décadas y haberse creado a miles de kilómetros. Esta forma de tecnificar y racionalizar la práctica de la intervención social afecta considerablemente al Trabajo Social (Modonesi, 2010), y lleva mucho tiempo estructurándose e imponiéndose a través de modelos de desarrollo únicos y transferibles desde países occidentales al resto del mundo (Escobar, 1992), en aras de un "progreso" (Chomsky, 1995) evaluado desde los parámetros de las grandes corporaciones, la acumulación de capitales (simbólicos y materiales), la naturaleza al servicio de seres humanos (cueste lo que cueste) y el consumismo como forma de vida.

Esta visión homogenizante no solo afecta a los niveles estructurales, también implica a las personas, negando las divergencias y simplificando, o directamente omitiendo, las diversidades. Este escenario nos sitúa en parcelas estanco, lo que puede parecer contradictorio: los individuos somos aglutinados y reducidos en categorizaciones funcionales, en paquetes que no se confunden nunca. Bajo este supuesto el trabajador social no es participante de programas o servicios sociales, no es población, o no es sujeto vulnerado en sus derechos, y los participantes en los programas no saben y no conocen cómo funcionan los (propios) problemas.

De este modo, el mundo del Trabajo Social se ha ido configurando de dos maneras: como profesionales (los técnicos racionales) y como "usuarios" (los irracionales que tienen que aprender a ser racionales). Cuando los "usuarios" no encajan en los perfiles "deseables" como "buenos usuarios" quedan relegados, y referidos como "obstáculos" del trabajo: "problemáticos", “difíciles", "negligentes", “no adherentes”, “disruptivos”, "especiales” y un sin fin de etiquetas lo que nos permite situarlos al margen de los márgenes.

En esta estructura rígida de unos (profesionales) y otros (usuarios), se extiende la idea de que es difícil que ambos puedan ser un colectivo y estar juntos para discernir, en igualdad, un tópico o un problema: piensen, si no, ¿por qué no se busca desde los equipos una manera de hacer partícipes a las personas, a familias, y a los niños, de los análisis de situación, mal llamados análisis de caso?

En el reparto de "sitios" los trabajadores sociales están, entonces, por encima de los participantes de los programas, pero por debajo de otras disciplinas. Esto, como ya se ha señalado, tiene que ver con la separación como mundos distintos e irreconciliables entre lo teórico (el conocimiento) y lo práctico (el quehacer), en términos de teoría de la intervención; y en nuestra escasa capacidad de producir conocimiento de "abajo" hacia "arriba" (Barnechea-García y Morgan-Tirado, 2010; Cifuentes-Gil y Pantoja-Kauffmann, 2019; JaraHolliday, 2020). 
Ortega-Senet

Podemos quedarnos y hablar desde los márgenes del discurso, la Sistematización de Experiencias puede hacer eso. También podemos asumir el desafío de escapar y deconstruir los términos centro-periferia, dentro-fuera, desbaratar la rigidez establecida a nivel estructural para decidir y lograr apropiarnos de lo que nos atañe para ver dónde nos lleva.

\title{
2.2 De las resistencias creactivas o la fiesta de los que faltan
}

\author{
Si usted quiere que en lugar de la institución oficial exista otra institución que \\ pueda desempeñar las mismas funciones, mejor y de otro modo, usted está ya \\ cogido en la estructura dominante. (Foucault, 1993, p. 43)
}

Para salir un poco de la victimización que integra la subalternidad, les propongo acercarnos a un concepto que no surge de la teoría propiamente dicha, sino que lo acuñó un creativo social llamado Unai Reglero para denominar un movimiento urbano en una ciudad catalana, Tarragona, y que se vino a sumar al cuestionamiento vital que se hizo en el 2011 en España por parte de los "Indignados". El término surge de combinar acción y creación "Creacción".

Cuando hablamos de una "resistencia creactiva", apunta a una acción que no se produce de forma reactiva, es decir, respondiendo a un sentimiento de presión y opresión, sino que, a partir de la consciencia constructiva de la realidad ejecuta formas "alter-nativas" y no "contra-nativas de praxis social, para desbaratar relaciones de dominación e inequidad (Ortega-Senet, 2015). Esta forma de considerar la resistencia es tan proactiva, que quizás, incluso, pierde la lógica de resistencia.

Pero propongo esta forma de pensar las acciones porque me permite retomar la conexión con la Sistematización de Experiencias y hacer una (re)vuelta a lo ontológico del Trabajo Social, a quiénes somos y qué somos, a través de un proceso de subjetivización alternativa a la subjetivización neoliberal que nos ha instrumentalizado.

Sabemos que las relaciones de poder en democracia no se desarrollan de forma impositiva, coercitiva, sino que se manifiestan como "un carácter desigual de las fuerzas que pugnan por imponer sus significados y revela los mecanismos sutiles mediante los cuales, se construyen las formas hegemónicas de significación y sus prácticas" (Foucault, 2009, p. 26). Es justamente nuestra capacidad de agencia (Bourdieu, 1994) la que nos permite una vía de escape de la subalternidad y de la reproducción de relaciones de poder que hemos venido señalando: si estas relaciones se construyen, también se deconstruyen (Guattari y Rolnik, 1999). Para ello se requiere acciones de cambio de lo que Dagnino denomina "política cultural", es decir, que se realice una redefinición de lo que es lo político y el espacio donde se pueden transformar las dinámicas poderativas (Dagnino, 2004). 
La política cultural, entonces, se presenta como un concepto totalizador que llama la atención sobre el vínculo constitutivo entre cultura y política, en las relaciones desiguales y las luchas por transformarlas, en la determinación de significados de las prácticas sociales y quién tiene el poder para definirlas; en la subjetividad y la identidad, ya que estas juegan un rol crucial en la aceptación o el rechazo de las relaciones de poder existentes. Es en definitiva una definición "enactiva". (Escobar, 1999, p. 134)

La enacción es un término que tiene mucho sentido con la Sistematización. Vamos a ver por qué. Si asumimos a la sociedad y sus relaciones como entramados en las redes cotidianas (Escobar, 1999) podemos observar la diversidad de intereses de la población, su heterogeneidad; concebirlas en conexión con sus procesos históricos, estructurales, sus resistencias y las estrategias ante las desigualdades. Mirar al colectivo en movimiento, o en pensamiento, puede llegar a conectarnos con lo que Jo Rowlands denominó el "poder con", es decir, el poder como elemento compartido. Lo que significa que las prácticas cotidianas están vivas y vinculadas a la definición ontológica del mundo de las relaciones humanas y son procesos constitutivos que pueden llegar a redefinir el poder social de manera clara o de manera tácita (Escobar, 1999).

A partir de lo anterior, es importante que hagamos una recapitulación de nosotros mismos. Me refiero a deconstruir esa idea de que los trabajadores sociales no somos parte de la comunidad, y que sí estamos más o menos implicados, vitalmente, en la mayor parte de los problemas que afectan a las personas con las que trabajamos. Esto supone una desotrorización para hacer una nos-otrorización con las personas que viven las situaciones problema. E implica subirnos al mismo barco, o siguiendo la metáfora estrella que nos acompaña a lo largo de este escrito, saber que estamos en el mismo baile. Esto también tiene que ver con recuperar la utopía, no en términos de imposible, sino en términos de posibles, transformando los sentidos comunes y lo que puede o no puede ser (Carañana, 2016; Ortega-Senet, 2017).

Para ello, es necesario liberarnos de la subjetividad gobernada, y generar nuestra propia subjetividad, ser capaces de enunciarnos de forma alternativa (Guattari y Rolnik, 1999). Estoy convencida de que instalar la autobservación, sea de la forma que sea, nos ayuda a crear autonomía, que a su vez permite reconstituir nuevas subjetividades e instalar esa idea de que toda praxis son historias siempre inacabadas, en las que los procesos son tanto o más importantes que los resultados.

La esperanza de enunciar futuros posibles, proyectos de cambio y utopías de cambio se conecta con la consciencia plena de lo que estamos haciendo y por qué lo estamos haciendo. El sentido común que surge de la experiencia compartida, valorada por los protagonistas, puede ser conseguida a través de muchos medios; y Sistematizar investigativamente las experiencias de intervención y acción social, colectivamente, es también uno de ellos. Nosotros lo estamos intentado. 


\title{
2.3 De cómo intentar que el baile llegue a la Academia y no al revés
}

La Sistematización de Experiencias tiene unas bases claras que se articulan a, y vamos a seguir aquí a Torres-Carrillo (2019): El reconocimiento de la complejidad de las prácticas de acción social; la reconstrucción de las prácticas desde la experiencia de sus protagonistas; la interpretación crítica y transformadora, el fortalecimiento emancipador de la práctica y el aporte “a la conceptualización de la transformación social y al pensamiento" (p. 87). El punto más controversial es quizás el último que señala Torres-Carrillo (2019):

\begin{abstract}
Los conocimientos generados a partir de un proceso de sistematización contribuyen a ampliar la comprensión que se posee sobre uno o varios campos, por ejemplo, la formación de maestros, las organizaciones sociales, los programas de acompañamiento, entre otros. El análisis de varias sistematizaciones sobre un mismo campo de prácticas puede generar reflexiones teóricas de mayor amplitud. Así mismo, la sistematización busca contribuir a la tradición de pensamiento crítico, generando categorías, claves interpretativas y reflexiones acerca de los sentidos y potencialidades de las prácticas de transformación social. (p. 87)
\end{abstract}

Y la controversia emana cuando debemos poner los límites a una institución que lidera la objetivación del conocimiento, la cuantificación de cualquier cosa como medio de validación, y la fragmentación de las disciplinas (a pesar del discurso interdisciplinario que surge siempre de ella).

Hay toda una discusión sobre hasta qué punto la articulación teórico-práctica desde la academia puede convertir nuestro pasacalle en un baile de salón. Es decir, si las estructuras rígidas de la academia en los procesos de sistematización que se realizan en las universidades, o desde las universidades, en los procesos de formación de estudiantes, puede influir de tal manera como para vaciar a la Sistematización del sentido original. Y ese cuidado por el sentido original, no es por un conservadurismo dogmático o romántico, sino por un más que fundado miedo a que las instituciones deformen e imbuyan de Racionalidad y Tecnificación el proceso de recogida y análisis de las experiencias.

También quiero ser cautelosa, porque todavía no sabemos bien en qué nos estamos convirtiendo como experiencia en nuestra propuesta particular de asignatura en la Universidad; aunque nos estamos esforzando en desarrollar una formación que permita generar experiencias interesantes para las estudiantes y las personas con las que comparten desafíos en sus prácticas profesionales o sus espacios personales de acción social, estamos recién comenzando nuestra cuarta versión, con pandemia por medio, y todavía queda mucho por andar. 
Ortega-Senet

\section{De la experiencia de la UCSC con la Sistematización Investigativa de Experiencias}

En la UCSC, llevamos apenas 4 años desde que comenzamos con la propuesta de Sistematización de Experiencias con estudiantes de Trabajo Social (la mayoría mujeres), la cual arrancó con la firme convicción de que a sistematizar se aprende sistematizando, como bien señala Cifuentes-Gil (1999). Todavía estamos inacabados, como debe ser, pero estamos intentando dar respuesta a una propuesta de formación que acoja el problema del conocimiento y la episteme política y ética.

Para ello, en tercer año, se realiza una asignatura obligatoria sobre Sistematización que les ofrece una introducción más bien teórica de qué es y qué no es; y, encuarto, tienen un optativo de Diseño de Sistematización (ya que pueden elegir entre este y el diseño de una Investigación Social) que, generalmente, culmina con una Memoria de grado para la obtención del título en quinto año. Revisaremos aquí la propuesta de Diseño ( $4^{\circ}$ año) y Memoria de Sistematización ( $5^{\circ}$ año) que es la que yo misma coordino.

Generalmente, se realiza una Sistematización articulada a sus espacios de prácticas, equipos y participantes de los servicios, pero también pueden elegir realizar la Sistematización en otros espacios de experiencia personal, como los lugares donde desarrollan sus activismos políticos, sus voluntariados o acciones sociales.

\section{Cuarto año, segundo compás}

Durante ese primer semestre, en un Primer Momento, los estudiantes realizan la justificación, la delimitación del foco y los ejes de interés conversando y reuniéndose con los colectivos y personas que se van a ir integrando en el trabajo. La delimitación del estudio de la intervención no es simple, dado que la conexión sistémica de las prácticas profesionales dificulta poner los límites. Generalmente, dice Castañeda (2014), la sistematización debe responder a preguntas clave que van a centrar la investigación en todo lo que se produce en relación a sus ejes de interés.

Este momento, en la medida de lo posible, busca hacer un trabajo constructivo y polifónico desde sus propias vivencias y conseguir reflexiones profundas que integran tanto lo vivido a nivel de ideas y hechos, como de emociones y sentimientos. No obstante, las estrategias para llevarlo a cabo van a depender de las posibilidades, los tiempos y la implicación de sus supervisores y profesionales del espacio de prácticas.

En Segundo Momento se desarrollan parte de los contextos que enmarcan el espacio de acción, lo que les permite, a su vez, familiarizarse con algunos elementos articulados a sus prácticas a su etapa pre-profesional, estos son: 
- $\quad$ El contexto legislativo: donde se señalan los elementos normativos y legales más importantes relacionados con la intervención y sus actores.

- $\quad$ El contexto de Política Social: donde se identifican planes, marcos generales del Programa en el que trabajan, dependencias políticas y administrativas, así como qué lugar ocupa la intervención en la red programática y objetivos de la política pública.

- El Contexto Institucional: donde se identifica la institución que gestiona la acción, si es que corresponde, cuál es su funcionamiento y objetivos, misión, visión y sello y todo aquello que les permita comprenderla.

- $\quad$ El Contexto Programático o acción social: En este apartado se describen, de forma muy general y orientativa, en qué consiste concretamente el Programa o actividad que acoge la experiencia interventiva, activista o praxis que se va a sistematizar.

El Tercer Momento es uno de los más importantes del primer semestre. Consiste en seleccionar las actividades mediante la cuales van a recoger la experiencia. Estas pueden ser tanto individuales como grupales, según los objetivos y las personas, aunque generalmente se relacionan con estas posibilidades:

- Técnicas para la reconstrucción (revisión de registros reflexivos, revisión de bitácoras o diarios de campo, técnicas de reconstrucción histórica o de hitos).

- $\quad$ Técnicas de observación directa y participante.

- $\quad$ Técnicas grupales (Flujograma, Café del Mundo, entrevistas grupales).

- Técnicas individuales (entrevistas abiertas, entrevista reflexivas).

- $\quad$ Técnicas audiovisuales (video voz, foto texto, realizadas con TIC).

- $\quad$ Revisión documental y registros.

En la asignatura tenemos dos técnicas estrellas: la Línea de Tiempo y el Flujograma. La Línea de Tiempo (Jara-Holliday, 2018) les permite reconstruir temporalmente recogiendo hitos y emociones adscritos a ellos de forma conjunta. El Flujograma (Ganuza, Olivari, Paño, Buitrago y Lorenzana, 2010), por su parte, facilita una identificación colectiva de nudos críticos y/o fortalezas (se puede hacer en negativo o en positivo) y las relaciones causa efecto 
Ortega-Senet

que se establecen entre ellos. La técnica también posibilita identificar el alcance de influencia que tienen los participantes sobre dichos nudos, y cuáles son los otros actores involucrados no presentes (Figura 1). Ambas técnicas son simuladas en clase con el propio grupo antes de ponerlas en práctica solas. A veces, incluso la entrenan en otros espacios donde se sienten más seguras antes de realizarlas con los colectivos participantes en la Sistematización; una situación que en realidad se conecta con las contradicciones y los roles que deben jugar las estudiantes en los espacios de práctica preprofesional (Parola, 2020).

Tras poner en ejecución las técnicas comprometidas, hay un Cuarto Momento en el que transcriben el material obtenido y, con ello, finalizan el primer semestre.

Figura 1. Ejemplo de diagrama de resultados del Flujograma.

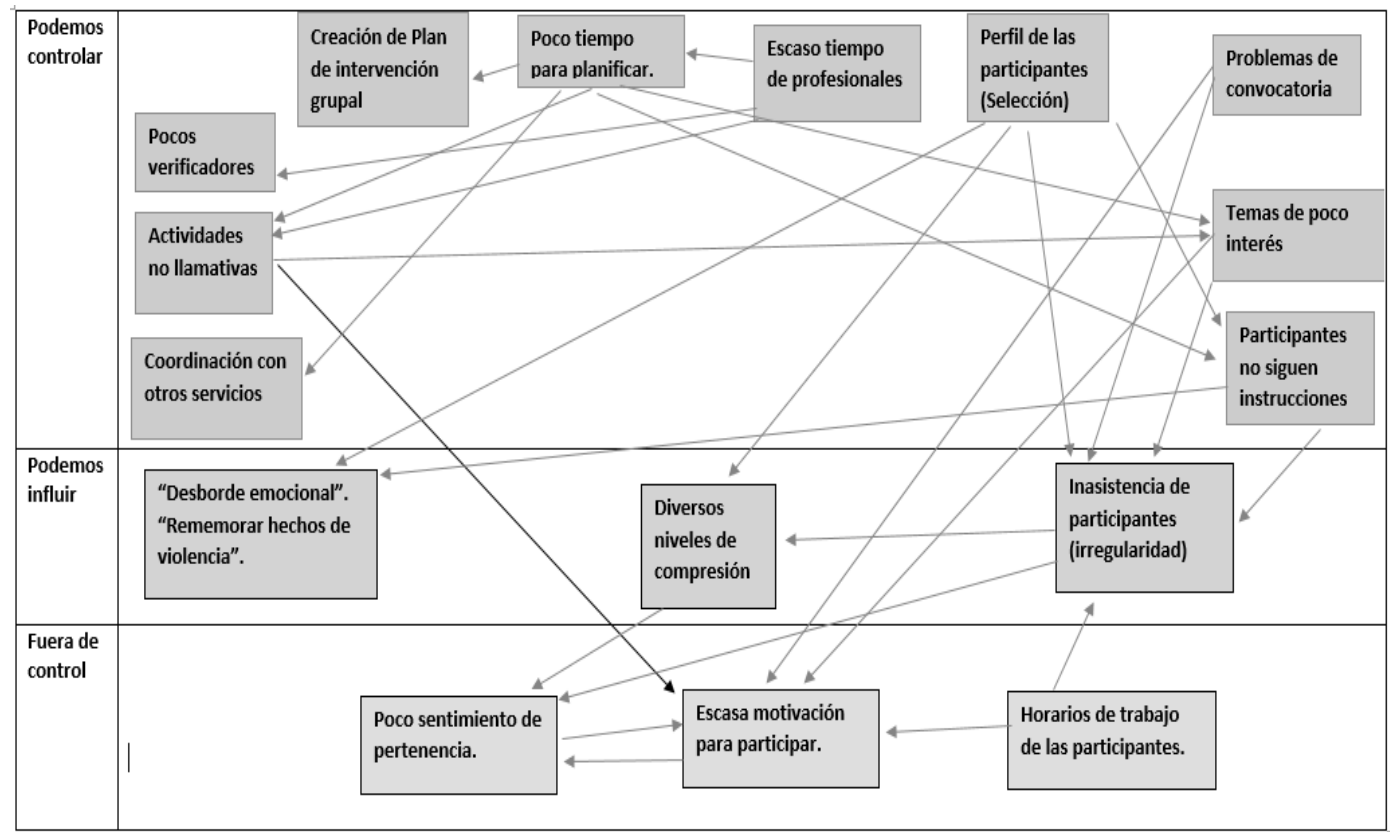

Fuente: Aranda (2019), Memoria de Sistematización “Experiencia de intervención social con mujeres sobrevivientes de violencia en Grupos de Apoyo del Centro de la Mujer Talcahuano-Hualpén".

\section{Quinto año, tercer compás}

En el segundo semestre, en el quinto año, la información recabada en el primer semestre es analizada en la asignatura de Memoria de Sistematización, para finalmente socializar este análisis con los participantes en un rito final que llamamos la" Retroalimentación". Esto se hace en espacios que los participantes prefieran, generalmente en los habituales para ellos, y este año, 2020, a través de plataformas online. Durante este último semestre, las estudiantes cuentan con un profesor guía individual que les ayuda en esta etapa, además de seguir contando con el grupo de compañeros de la asignatura. 
Ortega-Senet

Este semestre implica no solo los análisis, sino también finalizar otros aspectos de la contextualización y revisar "el estado del arte", constituyendo un Quinto Momento de recogida de información para completar los contextos:

- El contexto Paradigmático: el cual revisa filosofías, enfoques y paradigmas a los que se adscribe explícitamente la institución o el programa o la organización ejecutora en el que se producen la práctica.

- $\quad$ El contexto Socio-territorial: el que señala el alcance territorial de la institución y el programa. De este modo, se evidencian los alcances prácticos de la praxis sistematizada y la procedencia de los participantes. Así como procesos sociales directamente relacionados con el contexto del objeto de sistematización que influyen y permiten comprender por qué hoy día un territorio tiene unas características determinadas

- Contexto Histórico de la intervención: Este les complica bastante a los estudiantes, pero la idea es recoger los procesos históricos y dialécticos de formación y conformación de la comunidad en la que se interviene, hitos históricos y elementos esenciales para comprender la comunidad/territorio de hoy día.

- Y por último, la información de los Sujetos Participantes de la praxis: Esta parte solo tiene sentido si es una intervención social, porque en ella se identifica la población que generalmente acude y su relación con el Programa; es lo que en la investigación sería el universo, y se pueden señalar algunas de sus características particulares o posibles perfiles. Este ítem no debe confundirse con los Colectivos Implicados, que es el momento en el que describimos a las personas que efectivamente van participar de la Sistematización y se explica en la Metodología cuando se diseña el Proyecto.

Como antes se señalaba, en esta etapa también les pedimos que lean algunas Sistematizaciones e Investigación Acción Participantes o similares ya publicadas que tengan conexión con los ejes o los Programas con los que están trabajando. Esto les permite centrarse un poco y comprender mejor cómo desean seguir encaminando la tarea de análisis de resultados.

Lamentablemente, este Sexto Momento, el momento de organizar los resultados y el análisis, se realiza sin participantes ni equipos. No es lo ideal, pero las circunstancias no nos han permitido, hasta ahora, mayor participación en la segunda parte del trabajo. Este es uno de los nudos críticos de nuestra propuesta, entre algunos más que señalaremos un poco más adelante. 
Ortega-Senet

La etapa de análisis de resultados es quizás la más compleja. Por un lado, porque la estudiante no tiene a las personas (generalmente han terminado sus prácticas,) y porque se les pide un análisis profundo y crítico. Para ello, nos guiamos según lo que plantea Jara en su último artículo en el que sostiene que hay un momento de

distancia de sus propias experiencias para analizar e interpretar críticamente mirando aspectos del problema encontrados durante la reconstrucción y la recuperación de la experiencia, identificando categorías de análisis e interpretaciones teóricas relevantes, diseñando conclusiones y aprendiendo a obtener desde estos análisis e interpretaciones aportes generalizables y tópicos. (Jara-Holliday, 2020)

Fieles a nuestra apuesta por la búsqueda de un conocimiento situado, no asumimos la parte "generalizable" a la que alude Oscar Jara, y procuramos observar los límites de lo que pueda surgir. De este modo, a raíz de la información recogida se revisan las ideas de dos formas principalmente:

1. Una forma centrada en las personas, siendo fiel a lo que expresaron durante los procesos participativos. Este apartado conlleva un análisis básico desde la subjetividad de los actores que consiste en un análisis abierto y otro focalizado que nos permita ir agrupando la información en relación a los ejes.

2. Otra forma que se acoge es un análisis crítico que interpela a las tensiones, las contradicciones, las ausencias y cuestiona las implicancias de la acción.

Ambas visiones son sometidas a un análisis axial como lo sugieren Charmaz (2006) y Castañeda (2014), relacionando las diversas categorías que han ido surgiendo, y respondiendo a las preguntas básicas que movieron el trabajo. Estas categorías tienen diversas naturalezas:

a. Categorías prácticas: palabras e ideas que surgen de la práctica interventiva. Pueden ser metodologías, técnicas, estrategias, comunicación, organización, etc., elementos de la praxis en general.

b. Categorías ético-políticas: paradigmas, ideologías, creencias, relaciones de poder, discursos, categorías y performatividad para la constitución de relaciones tanto verticales como horizontales.

c. Categorías emocionales: sentimientos que se articulan en los procesos de relación humana y de intervención.

d. Categorías conceptuales: aquellas palabras e ideas que recogen significados teóricos y conceptuales desde la propia práctica. 
Ortega-Senet

Este último proceso del análisis conlleva y demanda lectura, teoría, y se compara con los hallazgos en otras experiencias; así como también se pueden explicar las ideas novedosas. Hay muchas posibilidades, y ese proceso es apoyado en la asignatura, practicando con información que las mismas estudiantes traen a clase.

El Séptimo Momento corresponde a las reflexiones de la experiencia, los aprendizajes y propuestas: En esta fase, las estudiantes describen su experiencia en relación al proceso de Sistematización y recogen los principales aprendizajes que han surgido del mismo. Seguidamente, respecto a ello, hacen propuestas de mejoramiento. Estas propuestas pueden ir encaminadas a: i) la praxis, el programa mismo, la institución y/o la política pública implicada en el tema; ii) a la formación universitaria; iii) nuevos focos de sistematización para profundizar o complementar lo realizado, o cosas que han quedado pendientes.

El último, el Octavo Momento, quizás el más emocionante, es la Retroalimentación. Los participantes son convocados, y aunque no siempre están todos, se procura que haya representación. Este es el momento en que los análisis, los aprendizajes y las propuestas son contrastadas y compartidas con los actores sociales. Los participantes pueden preguntar, indicar acuerdos y desacuerdos y aportar nuevas ideas. En esta instancia, las personas suelen recordar el proceso vivido y les sirve como espacio de reconsideración y construcción colectiva, por lo que, a menudo, surgen nuevas ideas y propuestas. Este es uno de los mejores momentos para todos y todas $\mathrm{y}$, en ocasiones, muy emotivo. Por lo general, es un momento muy grato que sirve de despedida a la estudiante de su práctica profesional, la cual le va aportando y, eso, le da mucha satisfacción. Este año se hizo a través de plataforma online, y contrariamente a lo que habíamos pensado, si bien es cierto que no ha sido un espacio muy cálido, resultó igualmente interesante y agradable. En la Tabla 1 se muestra la estructura por momentos, de los dos semestres que corresponden a las Asignaturas Diseño de Memoria de Sistematización Social y Memoria de Sistematización Social, que conforman un solo proceso: 
Ortega-Senet

Tabla 1. Estructura de los Momentos de trabajo de todo el ciclo (Diseño y Memoria)

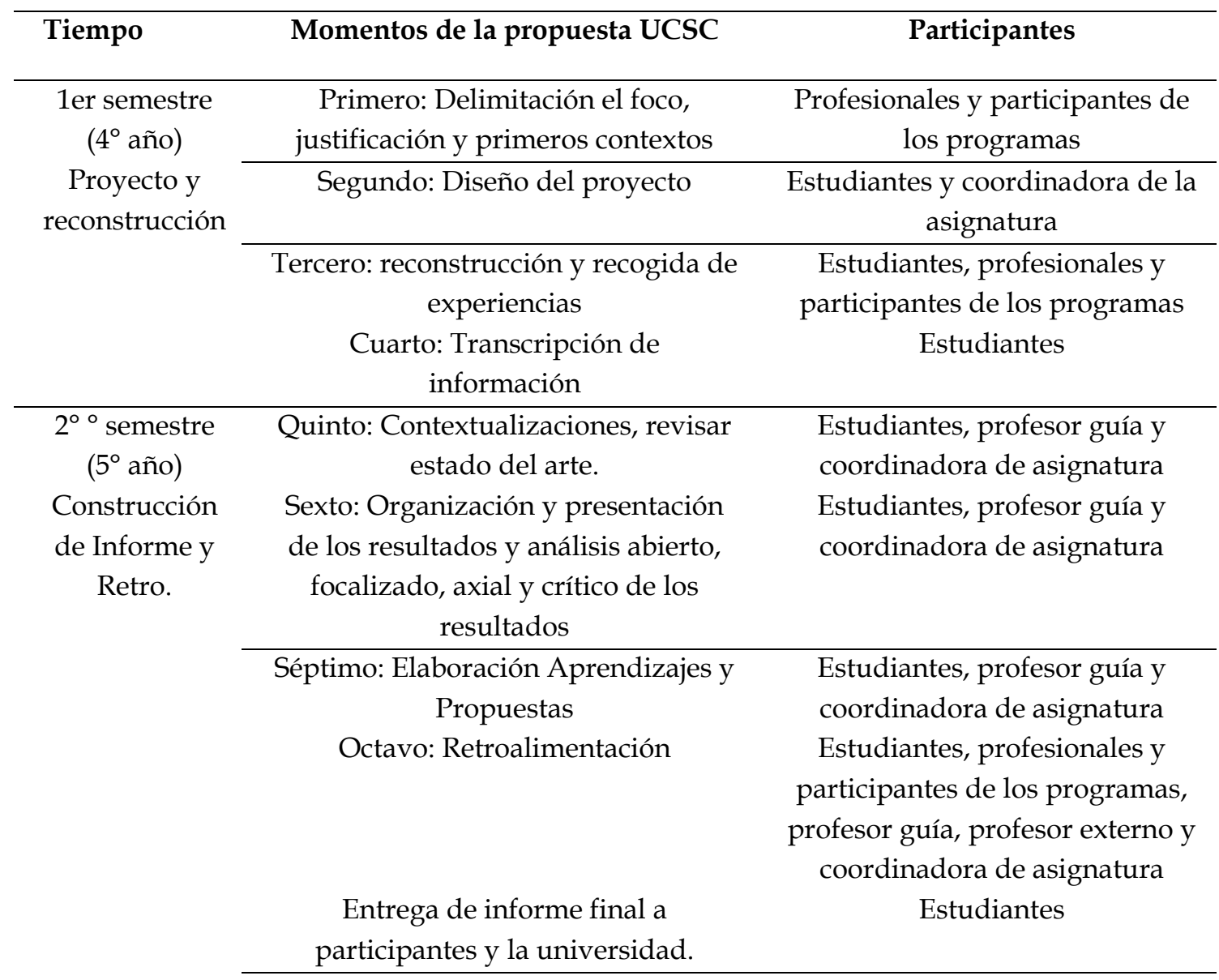

Fuente: elaboración propia.

\section{De los aprendizajes de la propuesta}

La propuesta de la UCSC tiene diversas aristas. En la última evaluación de la asignatura con las estudiantes y los profesores acompañantes, revisamos fortalezas y debilidades de la idea con la preocupación de mejorarla para el próximo año. Entre algunos nudos críticos quiero resaltar los siguientes:

- $\quad$ El hecho de que las estudiantes hagan el proyecto en un semestre distinto y que los profesores guías se suman a un proceso que ya comenzó. Aunque se tiene cierto margen de maniobra, los profesores no se sienten parte desde el principio.

- La "soledad" en los análisis. Si bien cuentan con los tutores, deben interpretar la información sin las personas participantes, lo que en un comienzo les supone todo un desafío, independientemente de que hayan surgido bastantes reflexiones en 
Ortega-Senet

las técnicas grupales. Pero suelen ir tomándole el pulso conforme lo desarrollan $\mathrm{y}$ algunas estudiantes igual se las ingenian para mantenerse comunicadas con algunos actores.

- Una estructura de los Momentos previos relativamente precisos que, si bien también ha sido vista como una fortaleza, por la claridad, puede ser limitante para aquellos que quieran hacer de otra manera. No obstante, hay que decir que todas hasta ahora han sido diferentes y cada una ha tenido un "sello" particular. Lo que consideramos, no es una mala señal.

- Algunas estudiantes han manifestado que, si bien terminan desarrollando capacidad crítica, se sienten abrumadas ante el trabajo de hacer una revisión más interpeladora.

- También evidencian que requieren más tiempo de apoyo individual, posiblemente porque es un proceso exigente.

- Por otro lado, también han relevado la importancia de los caracteres personales de cada una y cómo eso les puede jugar en contra: estudiantes más tímidas, muy autocríticas o inseguras, pueden sentirse sobrepasadas ante la exigencia de llevar la responsabilidad de la reflexión colectiva.

En relación a los elementos más positivos, en general estudiantes y profesores se sienten satisfechos al final; especialmente cuando los participantes les devuelven sus opiniones y complementan en la Retroalimentación. Hay estudiantes que se han referido al proceso como muy estimulante y que les "dan ganas de sistematizarlo todo", especialmente tras poner en práctica las técnicas de reconstrucción. De este modo, quiero mostrar algunas de las fortalezas señaladas:

- Las estudiantes sienten que efectivamente desarrollan la reflexión de la práctica, que les permite un cuestionamiento profundo y comprender cómo se lleva a cabo el trabajo y las acciones; sobre todo la aparición de elementos que normalmente les pasan desapercibidos.

- Por otro lado, han hecho hincapié en que el proceso les aporta muchos aprendizajes, a nivel intelectual y práctico.

- Resaltan también que es un proceso dinámico y que hace que todo pase muy rápidamente. Esto se relaciona con otras fortalezas señaladas, y es que es un proceso bien acotado, que cuenta con claridad respecto a lo que se espera de las estudiantes. 
- Las jóvenes dicen sentirse acompañadas y apoyadas por los profesores y visualizan espacios de contención tanto individuales como grupales.

- El hecho de estar articulado a su propia práctica o a sus participaciones sociales, también ha sido muy bien valorado. Ellas forman parte de la experiencia, lo que contribuye a que sea mucho más valioso.

- Si bien se ha hecho notar la estructura de momentos como limitantes, la propuesta en general se valora porque tiene un proceso de calidad (humana y material) en la reconstrucción y profundidad en los análisis. Esta ha sido una intención importante para nosotros. Estábamos preocupados de que no tuviera la complejidad necesaria para lograr trascendencia; pero, afortunadamente, ese no parece ser el problema, debido a que posee suficiente consistencia metodológica y analítica.

Todavía tenemos mucho por hacer, pero estamos contentas de lo que nuestra apuesta ha ido trayendo, incluida la publicación de una de las sistematizaciones por parte de una estudiante junto a su profesora (Gómez-Fernández y Cid-Arias, 2019), y la posibilidad de compartir y ampliar las redes con profesores de sistematización en otras Universidades. Todo eso nos motiva e ilusiona a seguir danzando.

\section{Conclusiones. Últimos pasos para ir despidiéndonos}

En fin, se ha intentado aportar a las cuestiones fundamentales teórico-prácticas de la sistematización, aunque solo es un granito. Torres-Carrillo (2019) nos invita a pensar en ello: “¿Qué tipo de conocimiento se produce desde la sistematización? ¿Cuál es el alcance epistémico de la misma? ¿Cuáles concepciones de práctica, experiencia y saber sustentan la sistematización? ¿Cómo participan los protagonistas de la práctica y sus saberes en la producción de conocimiento? (p. 84). Estas preguntas nos siguen interpelando a conversar y compartir, buscando maneras de colaborar en la construcción de "sujetos sociales críticos, reflexivos y conscientes de su potencial constructivo de los sistemas de los que hacen parte" (p. 84).

Una Sistematización corre el mismo riesgo, al igual que otras prácticas del quehacer en el Trabajo Social, de convertirse en un instrumento "objetivo" y "técnico", que procura desasirse de la dimensión ética-política. Es por eso que debemos estar especialmente alertas y vigilantes $\mathrm{y}$, más aún, cuando viene enmarcada desde espacios institucionales, como la universidad. 
Ortega-Senet

La idea es que la universidad termine siendo un espacio impregnando por las lógicas de la Sistematización de Experiencias, que vaya aportando, sumándose, al espíritu colaborativo y críticamente reflexivo, del que nunca debería dejar de ser parte. Analizar críticamente las experiencias es una propuesta provocativa en los términos que se han venido exponiendo en este texto. Las relaciones de poder se desmantelan; lo separado y parcelado se aglutina y comunitariza, y todo eso lo convierte en acciones de resistencia, capaces de construir nuevas subjetividades, autonomías y formas de ser y estar juntos en el Trabajo Social, ayudándonos a recuperar las utopías.

Cuando estas dinámicas lo permitan, generarán conocimiento, evidenciando saberes tácitos, desapercibidos (Caria, 2020), creando momentos conscientes individuales y acompañados, a estudiantes y a colectivos participantes, y poniendo en juego nuevas, otras, $\mathrm{u}$ ocultas, posibilidades epistémicas, generadoras de ideas y explicaciones con sentido común.

Agradecimientos: Gracias miles a los profesores que nos han acompañado hasta ahora, por aceptar el desafío de Sistematizar experiencias sin perder el Sur. Pero especialmente a las y los estudiantes de Memoria de Sistematización de la carrera de Trabajo Social de la UCSC por entusiasmarse con ella, de querer vivir el proceso "a concho" y disfrutar del camino.

A todos y todas, gracias por el baile.

\section{Referencias bibliográficas}

Abad-Miguélez, B., y Martín-Aranaga, I. (2015). El Trabajo Social ante la crisis. Nuevos retos para el ejercicio profesional de los y las trabajadores sociales. Cuadernos de Trabajo Social, 28(2), 175-185. Doi: 10.5209/rev_cuts.2015.v28.n2.48765.

Aranda, F. (2019). Memoria de Sistematización Experiencia de intervención social con mujeres sobrevivientes de violencia en Grupos de Apoyo del Centro de la Mujer Talcahuano-Hualpén (Trabajo de grado). Universidad Católica de la Santísima Concepción. Concepción, Chile.

Barnechea-García, M., y Morgan-Tirado, M. (2010). La sistematización de experiencias: producción de conocimientos desde y para la práctica. Tendencias $\mathcal{E}$ Retos, (15), 97-107.

Bermúdez-Peña, C. (2012). Intervención social desde el Trabajo Social: un campo de fuerzas en pugna. Prospectiva. Revista de Trabajo Social e intervención social, (16), 83-101. doi: 10.25100/prts.v0i16.1164. 
Ortega-Senet

Bermudo, J. M. (2001). Filosofía Política; luces y sombras de la ciudad. Barcelona: El Serval.

Bourdieu, P. (1994). Lenguage and simbolic power. Oxford: Polity Pre.

Carañana, J. P. (2016). Hacia un enfoque epistemológico pluralista en los estudios de comunicación y cambio: humanismo, ciencia y ecologismo. OBETS. Revista de Ciencias Sociales, 11(1), 129-164. doi: 10.14198/OBETS2016.11.1.06.

Caria, T. H. (2020). Os saberes tácitos dos trabalhadores profissionais. En L. Rangel y L. Sitja (Eds.), Mundo da Vida e Redes Educativas (pp. 1-23). Salvador: Editora da Universidade Federal da Bahia.

Castañeda, P. (2014). Propuestas metodológicas para trabajo social en intervención social y sistematización. Cuaderno Metodológico. Valparaíso: U. Valparaíso.

Charmaz, K. (2006). Constructing Grounded Theory: A Practical Guide Through Qualitative Analysis. London: SAGE.

Chomsky, N. (1995). Poder en el escenario Global. New Left Review, (0), 232-262.

Cifuentes-Gil, R. M. (1999). La sistematización de la práctica en Trabajo Social. Buenos Aires: Lumen.

Cifuentes-Gil, R. M. (2015). Construcción Disciplinar en trabajo social. Tendencias y Retos, 20(1), 51-80.

Cifuentes-Gil, R. M. y Pantoja-Kauffmann, G. F. (2019). Sistematización de experiencias para construir saberes y conocimientos desde las prácticas: Sustentos, orientaciones, desafíos. Argentina: Editorial Brujas.

Dagnino, E. (2004). ¿Sociedade civil, participação e cidadania: de que estamos falando? En D. Mato (Coord.), Políticas de Ciudadanía y Sociedad Civil en tiempos de globalización (pp. 95-110). Caracas, Venezuela: FACES, Universidad Central de Venezuela. Recuperado de http:/ / biblioteca.clacso.edu.ar/Venezuela/facesucv/20120723055520/Dagnino.pdf.

Escobar, A. (1992). El lugar de la naturaleza y la naturaleza del lugar: globalización o postdesarrollo. Antropología del Desarrollo. Barcelona: Paidós.

Escobar, A. (1999). El final del salvaje. Naturaleza, cultura y política en la antropología contemporánea. Bogotá: Cerec, Ican.

Foucault, M. (1993). Microfísica del poder. Madrid: La Piqueta.

Foucault, M. (2009). Nacimiento de la Biopolítica. Madrid: AKAL.

Ganuza, E., Olivari, L., Paño, P., Buitrago, L. y Lorenzana, C. (2010). La democracia en acción: una visión desde las metodologías participativas. Málaga: Antígona.

García-Martín, L. (2017). Vigilar y corregir: el trabajo social en las obras de Foucault y Donzelot. Cuadernos de Trabajo Social, 30(2), 357-367. doi: 10.5209/cuts.54585. 
Gianna, S., y Mallardi, M. (2011). Tensiones y contradicciones en la teleología de los procesos de intervención en trabajo social. Tendencias $\mathcal{E}$ Retos, (16), 17-31.

Gómez-Fernández, V., y Cid-Arias, F. (2019). Experiencia colaborativa de la Explotación Sexual Comercial de la Niñez en Biobío. Revista Perspectivas, (33), 11-172. doi: 10.29344/07171714.33.2052.

Guattari, F., y Rolnik, S. (1999). Micropolitca. Cartografias del deseo. Madrid: Traficante de sueños.

Harris, J. (2014). (Against) Neoliberal social work. Critical and Radical Social Work, 2(1), 7-22. doi: 10.1332/204986014x13912564145528.

Hicks, S. (2016). Theory and social work: A conceptual review of the literature. International Journal of Social Welfare, 25(4), 399-414. doi: 10.1111/ijsw.12215.

Jara-Holliday, O. (2018). La sistematización de experiencias: práctica y teoría para otros mundos posibles. Bogotá: CINDE.

Jara-Holliday, O. (2020). Systematisation of experiences: New paths to academic work in universities. International Journal of Action Research, 16(1), 62-74. doi: 10.3224/ijar.v16i1.05.

Modonesi, M. (2010). Subalternidad, antagonismo, autonomía. Marxismo y subjetivación política. Buenos Aires: CLACSO.

Muñoz-Arce, G. (2018). Contra la exclusión: Lugar de enunciación e intervención social en la primera línea. Polis, 17(49), 259-278. doi: 10.4067/s071865682018000100259.

Muñoz-Arce, G., \& Pantazis, C. (2019). Social exclusion, neoliberalism and resistance: The role of social workers in implementing social policies in Chile. Critical Social Policy, 39(1), 127-146. doi: 10.1177/0261018318766509.

Ortega-Senet, M. B. (2015). Trabajo Social como Transdisciplina: hacia una teoría de la intervención. Revista Cinta de Moebio, (54), 278-289. Recuperado de http://www.scielo.cl/pdf/cmoebio/n54/a05.pdf.

Ortega-Senet, M. B. (2017). El estudio y análisis de las intervenciones sociales consideradas como culturas políticas. Cinta de Moebio, (60), 286-294. doi: 10.4067/S0717-554X2017000300286.

Ortega-Senet, M. B., Gómez-Fernández, V., \& Tierney, E. M. (2020). Critical knots, tensions, and daily resistances in the work against commercial sexual exploitation of children: A reflection from Chilean practitioners. International Social Work. doi: 10.1177/0020872819899434.

Parola, R. N. (2020). Problematizando las prácticas preprofesionales en Trabajo Social. Desafíos y perspectivas. Prospectiva. Revista de Trabajo Social e intervención social, (29), 73-92. doi: 10.25100/prts.v0i29.8714. 
Saavedra-Vásquez, J. (2018). Intervención social como dispositivo discursivo. TS Cuadernos de Trabajo Social, (17), 1-13.

Torres-Carrillo, A. (2019). La sistematización como acción investigativa. En P. PañoYañez, R. Rébola y M. Suárez-Elias. (Comp.), Procesos y Metodologías Participativas. Reflexiones y experiencias para la transformación social (pp. 74-92), Uruguay: Cenur Litoral, Universidad de la República de Uruguay y CLACSO. Vivero-Arriagada, L. (2017). Desafíos de una práctica ético-política. El Trabajo Social chileno post-dictadura. Revista Katálysis, 20(3), 344-352. doi: 10.1590/198202592017 v20n3p344. 


\section{OTROS ARTÍCULOS DE PROSPECTIVA No. 31 DE 2021}

\section{PRESENTACIÓN}

Presentación. Reflexiones sobre desafios al publicar sistematizaciones

Rosa María Cifuentes-Gil

\section{EDITORIAL}

Reflexiones sobre Trabajo Social: aportes de la Sistematización

María Rocío Cifuentes-Patiño

\section{ARTÍCULOS}

Hacer lo que se sabe, pensar lo que se hace. La sistematización como modalidad investigativa Alfonso Torres-Carrillo

Aportes y desafios de la Sistematización de experiencias en el Trabajo Social y la extensión crítica. Apuntes y reflexiones desde la perspectiva de la Educación Popular

María Rosa Goldar

Valeria Chiavetta

La sistematización en Trabajo Social y la epistemología feminista del punto de vista. Diálogos sobre la producción de conocimiento sustentada en experiencias

Ruth Noemí Parola

María Florencia Linardelli

La Sistematización investigativa de las experiencias: del baile de los que sobran a la fiesta de los que faltan

María Belén Ortega-Senet

Sistematización y Trabajo Social en Chile. El largo y sinuoso camino

Patricia Lorena Castañeda-Meneses

Ana María Salamé-Coulon
Sentipensar la pandemia COVID-19 desde la sistematización de la experiencia en Trabajo Social: reflexiones del profesor Oscar Jara Holliday

Elia Sepúlveda-Hernández

La sistematización de experiencias, una investigación social cualitativa que potencia buenas prácticas de convivencia y gobierno. La experiencia de un conjunto residencial multifamiliar en Cali, Colombia Martha Lucia Echeverry-Velásquez Manuela Prada-Dávila

Construcción de subjetividades epistemológicaspolíticas de profesoras y profesores de Investigación social en una universidad privada y confesional en Bogotá

Giovanni Mora-Lemus

Sistematización de la experiencia Reconocimiento de los derechos humanos del adulto mayor en dos familias residentes en Cali y Valledupar (Colombia)

Lina María Cuello-Lacouture

Jimena del Pilar Jaramillo-Jaramillo

La memoria transformadora como estrategia de intervención profesional en los procesos de reconciliación social: comprensión a partir de mujeres campesinas, excombatientes y jóvenes en Manizales, Colombia

Yeimmy Stephania Corredor-Sotelo

Juliana Fuertes-Fuertes

Sistematización de una estrategia de educación informal implementada en personas privadas de la libertad en el establecimiento penitenciario de mediana seguridad y carcelario de Barranquilla, Colombia

Rafael Humberto Herrera-Mercado Rafael Alberto Zambrano-Vanegas 
Aportes significativos del proceso de intervención comunitaria con la Escuela Popular de Comunicación Alternativa Jaime Garzón de la ciudad de Cúcuta, Colombia

Carlos Lasso-Urbano

La sistematización de la intervención como metodología de investigación en Trabajo Social. Importancia práctica y teórica de la fase de recogida de datos en la intervención social según experiencia del Programa de Apoyo a las Familias en Zaragoza, España

Elisa Esteban-Carbonell

Nuria Del Olmo-Vicén

Papel de la sistematización de experiencias en los procesos de evaluación de intervenciones de salud pública en la Comuna Saludable por la Paz, Cali - Colombia

Jenny Faisury Peña-Varón

Paola Andrea Marín-Velásquez

Janeth Mosquera-Becerra

Experiencia de intervención social en hogares comunitarios integrales del barrio Alfonso Bonilla Aragón, Cali - Colombia

Julián Alexander Montaño-Cárdenas

Las políticas sociales y el gobierno de la "población indígena". Estrategias y regulaciones en el multiculturalismo chileno

Rodrigo Agustín Navarrete-Saavedra

Representaciones sociales sobre estilo de autoridad y tipos de interacción en cuidadores de residencias de protección infantil en Chile

Marcelo Gallegos-Fuentes
Carmen Gloria Jarpa-Arriagada

Reflexiones sobre inseguridad social y cuestiones penales. Una respuesta estratégica a partir de experiencias de cooperativismo con ex detenidos en Argentina

Analia Elizabeth Otero

Yael Yanina Barrera

Desarrollo y salud: la emergencia de un nuevo paradigma

Jesús María Sánchez-Ordóñez

Trabajo Social en ejercicio libre: la perspectiva profesional en España

Paula Frieiro-Padín

Tamara Fernández-Arias

Rubén González-Rodríguez

\section{RESEÑAS}

Social Work and the City: Urban Themes in 21stCentury Social Work

Felipe Saravia-Cortés

Respuestas del Trabajo Social ante emergencias sociales y problemáticas sociales complejas de México y España

Felipe Saravia-Cortés

El feminismo, el género y la profesionalización del trabajo social en Colombia (1936-2004)

Ambar Oriana Serna-Lombo

El puño invisible. Arte, revolución y un siglo de cambios culturales

Carlos Arturo Robledo-Marín

\section{PROSPECTIVA}

No. $31 \bullet$ ene.-jun. 2021

e-ISSN: 2389-993X • Universidad del Valle 